This is the pre-peer reviewed version of the following article:

Fra-Vázquez, A. , Pedrouso, A. , Palmeiro-Sánchez, T. , Moralejo-Gárate, H. and Mosquera-Corral, A. (2018), Feasible microbial accumulation of triacylglycerides from crude glycerol. J. Chem. Technol. Biotechnol, 93: 2644-2651, which has been published in final form at https://doi.org/10.1002/jctb.5618.

This article may be used for non-commercial purposes in accordance with Wiley Terms and Conditions for Use of Self-Archived Versions. 


\title{
Feasible microbial accumulation of triacylglycerides from crude glycerol
}

\author{
A. Fra-Vázquez ${ }^{a}$, A. Pedrouso ${ }^{a}$, T. Palmeiro-Sánchez ${ }^{a}$, H. Moralejo-Gárate ${ }^{a}$ and A. \\ Mosquera-Corral $^{\mathrm{a}}$ \\ ${ }^{a}$ Department of Chemical Engineering, Institute of Technology, Universidade de \\ Santiago de Compostela, 15782 Santiago de Compostela, Galicia, Spain \\ * Corresponding author. Tel.: +34 881816739. E-mail address: andrea.fra@usc.es
}

\begin{abstract}
BACKGROUND: Crude glycerol, a by-product of the biodiesel production industry, was used to produce intracellular storage polymers for waste valorisation. The enrichment of a mixed microbial culture (MMC) in microorganisms with the ability to accumulate intracellular polymers was performed in a sequencing batch reactor (SBR) submitted to feast/famine conditions. The effect of different carbon sources in the accumulation of biopolymers was investigated.

RESULTS: A mixed microbial culture (MMC) enriched in yeast and bacteria was obtained using crude glycerol as feedstock. Accumulation experiments performed with crude glycerol, synthetic glycerol, and synthetic methanol, showed the feasibility of the MMC to produce different biopolymers.

Triacylglycerides (TAGs) accumulation up to $46 \mathrm{wt}$.\% in yeast cells was promoted by the presence of residual lipids in crude glycerol. However, bacteria from Betaproteobacteria class used glycerol mainly to accumulate $28 \mathrm{wt} . \%$ of polyglucose $(\mathrm{PG})$ and methanol as carbon source for cell growth.
\end{abstract}

CONCLUSIONS: As waste valorisation, a possible advantage which comes out of the present study is the use of open, non-sterile and non-defined systems to produce TAGs. These TAGs can potentially re-enter the biodiesel production process helping on the maximisation of the feedstock used in this process.

Keywords: Bacteria, Bioprocesses, Lipids, Recovery, Waste Treatment, Yeast 


\section{INTRODUCTION}

Biodiesel is a renewable fuel that emerged during the past decades as a potential alternative to petroleum. In Europe, its production capacity during 2014 was approximately 23 million tonnes (European Biodiesel Board, 2014). Biodiesel is obtained by transesterification of fats and oils, after the addition of an alcohol (methanol or ethanol) and a catalyst (like sodium hydroxide), which generates crude glycerol as a primary by-product. ${ }^{1}$ On weight basis, $1 \mathrm{~kg}$ of crude glycerol is obtained per $9 \mathrm{~kg}$ of biodiesel produced. This crude glycerol contains impurities such as water, methanol, inorganic salts and free fatty acids. ${ }^{2}$ Glycerol is widely used in the pharmaceutical, cosmetic and food industry. For these purposes, glycerol with high purity is required, which implies the application of several purification steps, ${ }^{3}$ increasing its production costs. Therefore, the development of an economical, efficient and feasible utilisation of the crude glycerol is needed to increase the profitability of the biofuel production process.

The conversion of crude glycerol into added-value products represents an interesting and innovative option to improve its usefulness. ${ }^{4-6}$ Besides appearing as a cheap carbon source, its high reduction degree offers the opportunity to obtain reduced chemicals at higher yields than those achieved with sugars. ${ }^{7}$ In particular, some microorganisms can use crude glycerol as carbon source to produce cytoplasmic inclusions such as polyhydroxyalkanoates (PHAs) and triacylglycerides (TAGs). ${ }^{8}$ PHAs, like polyhydroxybutyrate (PHB) and polyhydroxyvalerate (PHV), are polyesters also known as bioplastics that may become an alternative to conventional plastics due to their properties, with the advantage of being biobased and biodegradable. ${ }^{9}$ TAGs are 
accumulated as intracellular lipid granules, and they can be extracted and used as biodiesel feedstock, re-entering the production process and maximising the use of raw materials. $^{10}$

The intracellular lipid production is carried out by oleaginous microorganisms, which are defined as microorganisms with a microbial lipid excess of $20 \mathrm{wt} . \%$. Most of these processes are based on the use of pure cultures and comprise microalgae, fungi, yeast and bacteria. Microalgae grow very fast, and they are very rich in oil, accumulating a TAG content up to 70 wt.\%. ${ }^{11}$ Prokaryotic microorganisms also accumulate TAGs, ${ }^{12}$ but their average lipid content is lower (approximately 20-40 wt.\%). Some yeast strains are also considered as oleaginous microorganisms, ${ }^{13}$ as they can accumulate up to 70 wt.\%.

Technologies based on the use of mixed microbial cultures (MMC) have appeared as an alternative to pure cultures. These processes are carried out as open, non-sterile cultures, which allow the use of wastewater as feedstock. ${ }^{14,15}$ In general, biopolymer production with $\mathrm{MMC}$ is based on a three-stage process. The first unit is an acidifying reactor to produce volatile fatty acids (VFAs) from the raw wastewater. These VFAs are used as a substrate in a second unit to select accumulating microorganisms from a MMC by imposing a feast-famine regime. This strategy consists of alternating periods with presence (feast) and absence (famine) of carbon source in the liquid medium. Finally, the maximum accumulation capacity of the enriched $M M C$ is achieved in a third unit where nutrients (mainly nitrogen) are supplied in limiting concentrations and the organic carbon substrate is present in excess to promote the storage of polymer and prevent the biomass growth. The main advantage of using glycerol to produce 
biopolymers is that the acidification unit is not needed, with the consequent saving of operational costs. Most of the studies using crude glycerol as carbon source reported PHA production, mainly as the homopolymer PHB. Dobroth et al. ${ }^{16}$ obtained a low polymer yield ( $0.10 \mathrm{~g} \mathrm{PHB} / \mathrm{g}$ methanol) because they enriched a MMC in methylotrophic bacteria and only the methanol fraction of crude glycerol was used to accumulate PHB. However, Moita et al. ${ }^{17}$ observed a higher PHB content of 47 wt.\% with an enriched microbial community only consuming glycerol as carbon source to produce biopolymers. Moralejo-Gárate et al. ${ }^{18}$ also produced PHB up to $80 \mathrm{wt} . \%$, but they used a MMC that was previously enriched using synthetic glycerol as substrate. In the present study, the features of the biopolymer production carried out by a mixed microbial culture using crude glycerol (containing glycerol, methanol and residual lipids) as substrate were assessed. Firstly, the enrichment process of a mixed microbial culture with biopolymer-accumulating capacity was researched. Then, the maximum storage capacity of the enriched MMC and differences in the obtained biopolymers were evaluated in batch experiments performed using different carbon sources.

\section{MATERIALS AND METHODS}

\section{Sequencing batch reactor for biomass enrichment}

A double jacket tubular glass reactor with a working volume of $2 \mathrm{~L}$ was used to select a MMC with biopolymer production capacity. The system was operated as a sequencing batch reactor (SBR) under aerobic dynamic feeding (ADF) strategy to establish the alternate feast-famine conditions. The SBR operated in 24-hour cycles according to the following scheme (in minutes): feeding (0-6), reaction (7-1,429), effluent withdrawal 
$(1,430-1,435)$ and idle $(1,436-1,440)$ periods. At the end of each cycle, half of the total volume of the reactor was discharged and the same volume of feeding of the next cycle was supplied, which resulted in hydraulic and solid retention times (HRT and SRT, respectively) of 48 hours. The complete mixture of the reactor medium was achieved by air flow (6 L/min), which was introduced through a ceramic air diffuser located at the bottom of the reactor. The temperature was controlled at $30^{\circ} \mathrm{C}$ using a water jacket connected to a thermostatic bath. The $\mathrm{pH}$ was maintained at $7.0 \pm 0.5$ with a $\mathrm{pH}$ controller (Crison, Germany), adding either $\mathrm{NaOH}(0.5 \mathrm{M})$ or $\mathrm{HCl}(0.5 \mathrm{M})$. The dissolved oxygen (DO) concentration was measured with an oxygen pocket meter provided with a membrane sensor (Hach-Lange, USA).

Activated sludge collected from the municipal wastewater treatment plant of CaloMilladoiro (Spain) was used as inoculum. Crude glycerol was provided by a biodiesel production facility located in Begonte, Lugo (Spain) (Table 1). Glycerol, methanol and lipids content in this waste stream amounted to $66 \%, 20 \%$ and $11 \%$ of the total organic carbon (TOC). The alkaline $\mathrm{pH}$ and salinity of the crude glycerol corresponded to the presence of $\mathrm{NaOH}$, added as a catalyser for the reaction during biodiesel production. A volume of $1,000 \mathrm{~mL}$ of feeding was added at the beginning of each operational cycle, containing $100 \mathrm{~mL}$ of the carbon solution, $100 \mathrm{~mL}$ of nutrient solution and $800 \mathrm{~mL}$ of dilution water. The carbon solution contained $23 \mathrm{~mL} / \mathrm{L}$ of crude glycerol. The nutrient solution was: $\mathrm{NH}_{4} \mathrm{Cl}, 6 \mathrm{~g} / \mathrm{L} ; \mathrm{KH}_{2} \mathrm{PO}_{4}, 7 \mathrm{~g} / \mathrm{L} ; \mathrm{MgSO}_{4} 7 \mathrm{H}_{2} \mathrm{O}, 2 \mathrm{~g} / \mathrm{L} ; \mathrm{KCl} 1 \mathrm{~g} / \mathrm{L}$; and $20 \mathrm{~mL} / \mathrm{L}$ of trace element solution. ${ }^{19}$ Moreover, $1.5 \mathrm{~mL} / \mathrm{L}$ of a $33 \mathrm{~g} / \mathrm{L}$ allylthiourea solution (ATU) were added to the nutrient solution to prevent nitrification activity. 


\section{Batch assays to determine the maximum storage capacity}

The maximum biopolymer storage capacity of the enriched MMC was evaluated in batch experiments using biomass samples collected at the end of the operational cycles of the enrichment SBR (Table 2). Batch experiments were carried out under the same conditions as those applied to the enrichment reactor. However, only crude glycerol was dosed as carbon source, and no nitrogen source was supplied to force the conversion of the substrate into biopolymer and to prevent the biomass growth.

Different carbon sources were tested to evaluate their influence on the biopolymer production: crude glycerol (A1), synthetic glycerol (A2), a mixture of synthetic glycerol and methanol ( $75: 25$ ratio as $\mathrm{Cmmol}$ ) similar to the composition of the crude glycerol (A3) and a mixture of synthetic glycerol and methanol (25:75 ratio as $\mathrm{Cmmol}$ ) with the opposite ratio to that from the experiment A3 (A4). In all cases, approximately 500 Cmmol of substrate were added in one pulse at the beginning of the experiment.

\section{Analytical methods}

\section{Liquid phase}

Liquid samples were filtered through a mixed cellulose and ester filter of $0.45 \mu \mathrm{m}$ of pore size (Advantec, Japan) for the quantification of glycerol, methanol, total organic carbon (TOC) and ammonium. Glycerol concentration was determined by highperformance liquid chromatography (HPLC) with HP 1100 (Hewlett Packard, USA) using an Aminex HPX-87H-87H $300 \times 7.8 \mathrm{~mm}$ column (temperature fixed at $35^{\circ} \mathrm{C}$ ) coupled to a refractive index (RI) detector and $\mathrm{H}_{2} \mathrm{SO}_{4}(5 \mathrm{mM})$ as eluent. Methanol concentration was measured by gas chromatography (model 6850-FID, Agilent, USA) using a DB-WAX 
$30 \mathrm{~m} \times 0.25 \mathrm{~mm} \times 0.25 \mu \mathrm{m}$ column coupled to a flame ionisation detector (FID) and nitrogen as carrier gas. TOC concentration was determined by catalytic combustion (model TOC-5000, Shimadzu, Japan) with an automatic injector (model ASI-5000-S, Shimadzu, Japan). Ammonium and suspended solids, total (TSS) and volatile (VSS), concentrations were analysed according to Standard Methods. ${ }^{20}$

\section{Solid phase}

For the analysis of biopolymer content, fresh biomass samples were collected, and formaldehyde was added to stop the microbial activity. Then, samples were centrifuged, frozen and freeze-dried to obtain a solid phase. For TAGs and PHAs identification and quantification, the method proposed by Smolders et al. ${ }^{21}$ was applied. Commercial standards of TAGs (palmitic acid (PO500), oleic acid (01008) and linoleic acid (L1376)) and PHA (containing $88 \%$ of hydroxybutirate (HB) and $12 \%$ of hydroxyvalerate (HV)) were used (Sigma-Aldrich, USA). Freeze-dried biomass samples of 5 - $15 \mathrm{mg}$ and standards were weighed using an analytical balance (Mettler Toledo, USA) and transferred into borosilicate glass tubes. Volumes of $1.5 \mathrm{~mL}$ of dichloroethane, $1.5 \mathrm{~mL}$ of a solution of propanol (with $25 \% \mathrm{vol} / \mathrm{vol}$ of commercial hydrochloric acid) and $50 \mu \mathrm{L}$ of a solution of benzoic acid ( $1 \mathrm{mg}$ of benzoic acid in 100 $\mathrm{mL}$ of propanol) as internal standard were added. Tubes were heated for 4 hours at $100{ }^{\circ} \mathrm{C}$ and then cooled at room temperature. A volume of $3 \mathrm{~mL}$ of water was added to each sample and two phases (aqueous and organic) were formed. One $\mathrm{mL}$ of the organic phase was filtered over water-free sodium sulphate into vials. The propylesters were analysed by gas chromatography (Agilent, USA) equipped with a FID, on an HP Innowax column. For the extraction of polyglucose (PG), 5-15 mg of freeze-dried 
samples was weighed using an analytical balance (Mettler Toledo, USA) and transferred into borosilicate glass tubes. A volume of $4 \mathrm{~mL}$ of $\mathrm{HCl} 0.6 \mathrm{M}$ was added to each tube, and they were heated at $100{ }^{\circ} \mathrm{C}$ for 3 hours. Glucose was measured by the HPLC previously described above.

\section{Calculations}

Lipid concentration $(\mathrm{Cmmol} / \mathrm{L})$ in the liquid samples was estimated by the difference between the TOC concentration and the carbon corresponding to the addition of glycerol plus methanol concentrations in the sample. The biopolymer content of the biomass was calculated as a percentage of the measured TSS on mass basis according to equation [1]. The active biomass $(X)$ was obtained by subtracting the quantity of the stored compounds from the VSS, following equation [2]. The maximum specific glycerol and methanol consumption rates $\left(-q_{\mathrm{gly}},-\mathrm{q}_{\mathrm{MeOH}}\right)$ were estimated from the maximum slopes of the curves describing the evolution of the concentrations of these compounds, divided by the active biomass concentration $(\mathrm{Cmmol} /(\mathrm{Cmmol} \times \mathrm{h}))$. The same procedure was applied to calculate the biopolymer (TAG, PHB, PHV and PG) specific production rates $\left(\mathrm{q}_{\mathrm{TAG}}, \mathrm{q}_{\mathrm{PHB}}, \mathrm{q}_{\mathrm{PHV}}, \mathrm{q}_{\mathrm{PG}}\right)$. The yields $(\mathrm{Cmmol} / \mathrm{Cmmol})$ for TAGs $\left(\mathrm{Y}_{\mathrm{TAG} / \mathrm{TOC}}\right), \mathrm{PHB}\left(\mathrm{Y}_{\mathrm{PHB} / \mathrm{gly}}\right), \mathrm{PHV}\left(\mathrm{Y}_{\mathrm{PHV} / \mathrm{gly}}\right), \mathrm{PG}\left(\mathrm{Y}_{\mathrm{PG} / \mathrm{gly}}\right)$, and active biomass $\left(\mathrm{Y}_{\mathrm{X} / \mathrm{S}}\right)$ were obtained by dividing the specific production rate of each biopolymer by the consumption rate of the substrate.

$$
\begin{gathered}
\text { Biopolymer }(\%)=\frac{\text { g bipolymer }}{g \text { TSS }} \cdot 100 \\
\mathrm{Cmmol}_{X}=\mathrm{Cmmol}_{V S S}-\left(\mathrm{Cmmol}_{T A G}+\mathrm{Cmmol}_{P H B}+\mathrm{Cmmol}_{P H V}+\mathrm{Cmmol}_{P G}\right)
\end{gathered}
$$


The elemental composition of the active biomass was assumed to be $\mathrm{CH}_{1.8} \mathrm{O}_{0.5} \mathrm{~N}_{0.2}$.

\section{Microbiology}

The identification of active microbial populations was performed by applying the fluorescence in situ hybridization (FISH) technique. Fresh biomass samples were taken and the procedure described by Amann et al. ${ }^{22}$ was followed. Bacterial cells hybridised with the following probes: EUB338I, EUB338II, EUB338III, BET42a and EUK516 (Supplementary material, Table S.1). All probes were 5' labelled by fluorochromes FITC (Fluorescein-5-isocyanate) or Cy3 (Carbocyanine 3). Details on oligonucleotide probes are available at probeBase. ${ }^{23}$ DAPI (4, 6-diamidino-2-phenylindole) was used as universal dye for the detection of all DNA in the samples. Fluorescence signals were captured using an acquisition system (Coolsnap, Roper Scientific Photometrics) coupled to an epifluorescence microscope (Axioskop 2 Plus, Zeiss, Germany). Moreover, fresh samples were taken from the enrichment SBR to verify the biopolymer accumulation by lipid staining. Sudan IV solution was applied to fresh samples for $10 \mathrm{~min}$ and then, after the wash-out with distilled water to eliminate the excess of dye, safranin for 15 minutes. After drying, microorganisms were observed with a binocular vision microscope Axioskop 2 Plus (Zeiss, Germany).

\section{RESULTS AND DISCUSSION}

\section{Enrichment of a mixed microbial culture with crude glycerol}

The enrichment SBR was operated for 500 days using crude glycerol as feeding. The DO concentration was measured throughout the enrichment operational cycles to 
evaluate the selection of accumulating microorganisms. In the present research work, the end of the feast phase was considered when both glycerol and methanol were depleted, and its length decreased with the gradual enrichment of the system. During the first operational cycles, no significant changes in the DO profile were observed. However, in few weeks the feast-famine regime appeared accompanied by the substrate consumption. After 100 days of operation, the feast lasted 6 hours which corresponded to a feast/famine ratio of 0.25 . According to Dionisi et al. ${ }^{24}$, values of this ratio of approximately $0.40-0.50$ are associated to systems where biomass growth occurs, whereas values lower than 0.20 indicate the presence of cultures capable of biopolymer storage.

The progress of the MMC enrichment was also identified by changes in biomass and ammonium concentrations at the end of the SBR cycles (Figure 1). At the beginning of the operation, the biomass concentration was $0.3 \mathrm{~g}$ VSS/L. Then, this value increased and remained at approximately $0.7 \mathrm{~g}$ VSS/L from day 100 of operation onwards. In a similar way, ammonium consumption progressively increased, and its concentration

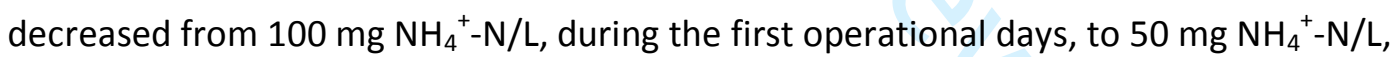
from day 100 onwards. This gradual decrease correlated with the increase of biomass concentration in the way that fitted with the theoretical nitrogen consumption for biomass growth. The stabilization of the value of the feast/famine ratio and the ammonium and biomass concentrations at the end of the enrichment cycles indicated that the MMC was enriched after 100 days of operation. The enrichment process was significantly longer than in previous studies using synthetic glycerol as substrate, which 
took 40 days. ${ }^{18}$ In the present study, the complex composition of the crude glycerol could slow down the enrichment of the microbial community.

Microbial analyses were performed to the enriched biomass on day 229 of operation. Two main microbial populations were identified: Eukarya and Bacteria (from class Betaproteobacteria). Large spherical cells corresponded to yeasts (Eukarya) (Figure 2A), whereas small cocci-shape microorganisms belonged to Betaproteobacteria (Figure 2B). Sudan IV staining confirmed the presence of biopolymers accumulated inside the yeasts as intracellular inclusions, which are presumably the TAGs. In some cases, they appeared as single granules and, in others, as several granules in the same cell (Supplementary material, Figure S.1).

\section{Characterisation of an enrichment cycle}

The performance of the enriched $\mathrm{MMC}$ was followed by measuring single operational cycles, which are represented by that of day 246 (Figure 3). Crude glycerol containing glycerol, methanol and lipids, which amounted to approximately $30 \mathrm{cmmol} / \mathrm{L}$ in total, was added as substrate at the beginning of the cycle. After crude glycerol addition, the DO concentration rapidly decreased during the feast period. During this period, its profile was characterised by the existence of two plateaus (Figure 3A). The first one lasted approximately 2 hours and corresponded to the oxygen consumption due to lipids and glycerol uptake. Then, the second plateau was coincident with the methanol depletion, which finished after 6 hours. The estimated glycerol specific uptake rate was $0.26 \mathrm{Cmol}_{g \mid y} /\left(\mathrm{Cmol}_{x} \cdot \mathrm{h}\right)$, four-fold the methanol specific uptake rate (0.06 $\left.\mathrm{Cmol}_{\mathrm{MeOH}} /\left(\mathrm{Cmol}_{\times} \cdot \mathrm{h}\right)\right)$. Lipid uptake rate was not calculated because lipids concentration 
was fully depleted immediately after substrate addition, presumably due to an initial adsorption phenomenon on the biomass surface. The lipids and glycerol consumption reverted in the accumulation of three different types of biopolymers during the first period of the feast: TAG, PHV and PG. The maximum accumulation percentages, reached in terms of cell dry weight, were of 25.5, 6.7 and 7.9 wt.\% for TAG, PHV and PG, respectively (Figure 3B). The compositions of lipids present in the crude glycerol and the TAGs accumulated as intracellular biopolymers were very similar (20\% palmitic acid, $30 \%$ oleic acid and $50 \%$ linoleic acid, wt.\%). These results, combined with carbon balance calculations, indicated that the lipids from the crude glycerol were uptaken and intracellularly accumulated as TAGs. Glycerol was used for biomass growth, which correlated with the decrease of ammonium concentration during this period, but it was also accumulated as small amounts of PHV and PG. After glycerol and lipids were consumed, both methanol and biopolymer consumption started. At the same time the biomass concentration increased, associated to growth on methanol and accumulated biopolymers.

Marang et al. ${ }^{25}$ observed a similar two-plateau DO profile using a synthetic mixture of acetate and methanol as carbon source in an enrichment reactor. They identified in the mixed culture two bacterial species: Plasticicumulans acidovorans as PHAaccumulating bacteria and Methylobacillus flagellatus as methylotroph organisms, which consumed acetate and methanol, respectively. They observed biopolymer production only when acetate was present, and simultaneous growth on methanol and PHA occurred during the second part of the feast phase. In the present research work, microbial populations from Eukarya and Bacteria were identified. It can be 
hypothesized that yeast were responsible for TAG accumulation, as these are recognized as the main storage component for energy in eukaryotic cells.$^{13}$ On the other hand, bacterial cells consumed glycerol and methanol to grow and produce PHV and PG. In this way, the coexistence of both microbial populations may provide this versatile behaviour of the MMC.

Few authors have previously worked on biopolymer production from crude glycerol. Moita et al. ${ }^{17}$ used crude glycerol as feedstock with a similar composition to the substrate used in the present study. They observed PHB and PG accumulation from glycerol, while methanol consumption was not complete. They obtained an enriched MMC with PHA-accumulating capacity but they did not observe TAGs production. Differences in the type of biopolymer accumulated may be due to the fact that these authors used a PHA-accumulating microbial culture previously acclimated to bio-oil as feedstock. However, in the present study activated sludge was used as inoculum for the MMC development. Dobroth et al. ${ }^{16}$ also obtained PHB as the main biopolymer but only utilising the methanol fraction of the crude glycerol as carbon source. Contrary to these results from literature, in the present study TAGs were found as the preferred storage compound when crude glycerol was supplied for the selection of a community with the ability to produce storage polymers. To the knowledge of the authors, TAG production from industrial wastes using a MMC had not been reported previously.

\section{TAG-accumulating capacity using crude glycerol as substrate}


The maximum accumulation capacity of the enriched MMC was evaluated using crude glycerol as substrate (experiment A1, Table 2). The concentration of crude glycerol was higher than that used in the enrichment SBR. Approximately $500 \mathrm{Cmmol}$ were added in one single pulse at the beginning of the experiment. No nitrogen source was added to force the complete conversion of substrate into biopolymers and prevent the microbial growth. However, nitrogen was present in the liquid media during the first hours of the experiment as residual ammonium coming with the sludge collected at the end of the operational cycle of the enrichment SBR.

After crude glycerol addition, lipids, glycerol and methanol concentrations evolved with a similar trend as in the enrichment cycle. Even though lipids were not analysed, the concentration was estimated from carbon balances considering measured glycerol, methanol and TOC. A sudden decrease of lipids was observed during the first 10 minutes of the experiment, presumably due to their adsorption on the biomass surface. During the feast phase glycerol was preferred over methanol and totally consumed in 7 hours after substrate dosage (Figure $4 A$ ). Only $24 \%$ of the added methanol was consumed during the same period. The corresponding specific substrate consumption rates were of $0.43 \mathrm{Cmmol}_{\text {gly }} /\left(\mathrm{Cmmol}_{\mathrm{x}} \cdot \mathrm{h}\right)$ and 0.13

$\mathrm{Cmmol}_{\mathrm{MeOH}} /\left(\mathrm{Cmmol}_{\mathrm{X}} \cdot \mathrm{h}\right)$, respectively (Table 3$)$.

The consumption of the carbon compounds present in the crude glycerol was accompanied by the production of TAG, PHV and PG (Figure 4A). TAGs were accumulated as the main storage compound up to $46.3 \mathrm{wt} . \%$ after 2.5 hours. Moreover, the extracted TAGs from the biomass samples composition was of 20 wt.\% palmitic acid, 30 wt.\% oleic acid and 50 wt.\% linoleic acid, very similar to the 
composition of fatty acids of the crude glycerol. PG was also determined as biopolymer, and its highest amount of 28 wt.\% was measured seven hours after substrate addition, which corresponded to the complete glycerol depletion. PHAs were measured as PHV and reached a low value of $7 \mathrm{wt} . \%$.

In the present research work, the presence of lipids in the crude glycerol presumably selected TAG-accumulating microorganisms in the enrichment SBR. Different strains of oleaginous yeasts have been previously reported as capable of the production of intracellular lipids from crude glycerol with maximum accumulations as dry weight content of 25 wt. $\%{ }^{26}, 32$ wt. $\%{ }^{27}$ and 64 wt. $\% .{ }^{10}$ The maximum TAG accumulation in the present study was $46.3 \mathrm{wt} . \%$, which is in the range of the obtained values in those previous studies with pure cultures.

\section{Biopolymer production with different carbon sources}

To determine the separated effects of the glycerol and methanol on the accumulating capacity of the enriched MMC, experiments with synthetic carbon sources were performed (A2-A4, Table 2). As in experiment $\mathrm{A} 1$ with crude glycerol, $500 \mathrm{Cmmol}$ were added in one single pulse and no nitrogen source was added to force the biopolymer accumulation.

Accumulation experiments with synthetic glycerol in experiment A2 (Table 2), indicated that the complete depletion of this carbon source occurred 22 hours after substrate dosage (Figure 4B). Compared with the previous experiment with crude glycerol, the accumulation of TAGs was very low (11.4 wt.\%). Moreover, with glycerol as substrate the enriched MMC could produce PG as the main accumulated 
biopolymer (54.3 wt.\%), but also 3.6 wt.\% and 14.4 wt.\% of PHB and PHV, respectively. In this case, the specific substrate consumption rate was of $0.55 \mathrm{Cmmol}_{\mathrm{gly}} /\left(\mathrm{Cmmol}_{\mathbf{x}} \cdot \mathrm{h}\right)$ (Table 3).

In the experiment $\mathrm{A} 3$, the composition of the crude glycerol was mimicked with a mixture of synthetic glycerol and methanol (75:25 ratio as $\mathrm{Cmmol}$ ) to evaluate the MMC accumulation behaviour (Table 2). Glycerol was consumed faster than methanol, with specific uptake rates of $0.48 \mathrm{Cmmol}_{\mathrm{gly}} /\left(\mathrm{Cmmol}_{\mathrm{x}} \cdot \mathrm{h}\right)$ and 0.09 $\mathrm{Cmmol}_{\mathrm{MeOH}} /\left(\mathrm{Cmmol}_{\mathrm{x}} \cdot \mathrm{h}\right)$, respectively (Figure $\left.4 \mathrm{C}\right)$. These values were very similar to those obtained in the experiment A1 with crude glycerol (Table 3). However, in this case, only PG accumulation was observed up to approximately $40 \mathrm{wt} \%$. As it occurred in experiment $A 2$, TAG accumulation did not take place.

A mixture of glycerol and methanol in a ratio of $25: 75$ as $\mathrm{Cmmol}$, respectively (experiment A4, Table 2), was used as substrate to evaluate the effects of the methanol on the MMC. Methanol concentration at the beginning of the experiment was higher than that of glycerol, but its consumption rate was significantly lower 0.17 $\mathrm{Cmmol}_{\mathrm{MeOH}} /\left(\mathrm{Cmmol}_{\mathrm{x}} \cdot \mathrm{h}\right)$ compared to that of the glycerol of $0.25 \mathrm{Cmmol}_{\mathrm{gly}} /\left(\mathrm{Cmmol}_{\mathrm{x}} \cdot \mathrm{h}\right)$ (Figure 4D). No significant accumulation of biopolymers was obtained in this assay. Small amounts of PHB and PG were measured, reaching the maximum dry weight content of 8.6 and 8.2 wt.\%, respectively, when glycerol was depleted. These results indicated that the glycerol was the most probable substrate used as carbon source to accumulate these two polymers. However, the PG production decreased in this experiment and it was nine times lower than in $A 2$ and five times less than in experiments $\mathrm{A} 1$ and $\mathrm{A} 3$. Moreover, contrary to the accumulation experiments with 
other substrates, ammonium was not completely consumed, and biomass continued growing throughout the whole cycle These results may indicate a possible inhibitory effect of methanol, as previous studies indicated that methanol inhibits the growth of PHB accumulating bacteria at concentrations above $0.5 \%$ (vol/vol). ${ }^{28}$ Accumulation experiments (A1-A4, Table 2) provided different results depending on the composition of the used carbon compounds. Glycerol uptake rate was similar in the experiments where large glycerol concentrations were added. Glycerol was preferably intracellularly accumulated as PG, as can be proven by the results of experiments A2 (synthetic glycerol) and A3 (glycerol:methanol ratio 75:25), where percentages were higher than $40 \mathrm{wt} . \%$ once glycerol was consumed. These results seemed to indicate that glycerol was the main carbon source for PG accumulation. Moralejo-Gárate et al. ${ }^{29}$ reported a yield of $0.40 \mathrm{Cmmol}_{\mathrm{PG}} / \mathrm{Cmmol}_{\mathrm{gly}}$ using synthetic glycerol, slightly higher than the value obtained in the present study $(0.33$ $\left.\mathrm{Cmmol}_{\mathrm{PG}} / \mathrm{Cmmol}_{\mathrm{gly}}\right)$. Both PHV and PHB were produced at low percentages, no higher than $14 \%$ and PHV as the preferred compound. However, in previous studies, PHB was obtained as the main biopolymer using both synthetic and crude glycerol. MoralejoGárate et al. ${ }^{18}$ obtained 80 wt.\% of PHB using synthetic glycerol. This is a very high value compared to those obtained with the same substrate in the present study of 4 and 15 wt. $\%$ of PHB and PHV, respectively. Dobroth et al. ${ }^{16}$ obtained 67 wt. $\%$ of PHB consuming only the methanol fraction, which corresponded to $40 \mathrm{wt} . \%$ of the total carbon supplied and it is higher than the methanol in the crude glycerol used in this study. With a similar crude glycerol composition, Moita et al. ${ }^{17}$ reached maximum PHB content of 47 wt.\%. However, they used biomass with PHA-accumulating capacity 
already acclimated to bio-oil as feedstock. In all these cases, the substrate composition (synthetic) or the MMC (acclimated microorganisms) differed from the crude glycerol used in the present research work. Therefore, the selected accumulatingmicroorganisms in the corresponding enrichments were expected to be different. The low PHA values might be attributed to the characteristics of the enriched MMC, where TAG-accumulating microorganisms were preferably selected instead of PHAaccumulating microorganisms. Only in the experiment with crude glycerol (A1) the enriched MMC could accumulate high TAG content, indicating that the presence of lipids is required to obtain significant intracellular TAG accumulation.

\section{CONCLUSIONS}

In the present study, a mixed microbial culture with biopolymer-accumulating capacity was enriched using crude glycerol as carbon source. Microorganisms from Eukarya and Bacteria domains were selected and cohabitated in the MMC, which accumulated a mixture of biopolymers (TAG, HB, HV and PG). Yeasts were mainly responsible for the lipids storage as TAGs, while bacteria used glycerol to accumulate the remaining biopolymers and methanol for growth. Maximum TAG accumulation of 46.3 wt.\% was observed only when crude glycerol containing lipids was used as substrate. When synthetic carbon sources were used, the maximum accumulation corresponded to PG, which indicated that fatty acids present in the crude glycerol promoted TAG accumulation.

The conversion of crude glycerol to lipids is important for waste valorisation. A possible advantage which comes out of the present study is the use of an open, non- 
sterile and non-defined enrichment, which is expected to lead to a significant decrease in the production costs due to lower energy requirements and simpler operation, in comparison with the available studies that operated under sterile conditions, using pure and defined cultures.

\section{ACKNOWLEDGEMENTS}

This research was supported by the Spanish Government (AEI) through FISHPOL (CTQ2014-55021-R) and GRANDSEA (CTM2014-55397-JIN) projects. The authors belong to the Galician Competitive Research Group GRC ED431C 2017/29 and to the CRETUS Strategic Partnership (AGRUP2015/02). All these programmes are co-funded by the FEDER (EU). 


\section{REFERENCES}

1. Gerpen JV, Biodiesel processing and production. Fuel Process Technol 86:1097107 (2005).

2. Samul D, Leja K, Grajek W, Impurities of crude glycerol and their effect on metabolite production. Ann Microbiol 64:891-8 (2014).

3. Johnson DT, Taconi KA, The glycerin glut: Options for the value-added conversion of crude glycerol resulting from biodiesel production. Environ Prog 26:33848 (2007).

4. Yang F, Hanna MA, Sun R, Value-added uses for crude glycerol: a byproduct of biodiesel production. Biotechnology for Biofuels 5:1-13 (2012).

5. Luo X, Ge X, Cui S, Li Y, Value-added processing of crude glycerol into chemicals and polymers. Bioresour Technol 215:144-54 (2016).

6. Anitha M, Kamarudin SK, Kofli NT, The potential of glycerol as a value-added commodity. Chem Eng J 295:119-30 (2016).

7. da Silva GP, Mack M, Contiero J, Glycerol: A promising and abundant carbon source for industrial microbiology. Biotechnol Adv 27:30-9 (2009).

8. Thomson N, Summers D, Sivaniah E, Synthesis, properties and uses of bacterial storage lipid granules as naturally occurring nanoparticles. Soft Matter 6:4045-57 (2010).

9. Chen GG-Q. Plastics from Bacteria. Chen GG-Q, editor: Springer Berlin Heidelberg; 2010. 
10. Cheirsilp B, Louhasakul Y, Industrial wastes as a promising renewable source for production of microbial lipid and direct transesterification of the lipid into biodiesel. Bioresour Technol 142:329-37 (2013).

11. Meng X, Yang J, Xu X, Zhang L, Nie Q, Xian M, Biodiesel production from oleaginous microorganisms. Renewable Energy 34:1-5 (2009).

12. Alvarez H, Steinbüchel A, Triacylglycerols in prokaryotic microorganisms. Appl Microbiol Biotechnol 60:367-76 (2002).

13. Sorger D, Daum G, Triacylglycerol biosynthesis in yeast. Appl Microbiol Biotechnol 61:289-99 (2003).

14. Ben M, Kennes C, Veiga MC, Optimization of polyhydroxyalkanoate storage using mixed cultures and brewery wastewater. Journal of Chemical Technology \& Biotechnology 91:2817-26 (2016).

15. Serafim LS, Lemos PC, Oliveira R, Reis MA, Optimization of polyhydroxybutyrate production by mixed cultures submitted to aerobic dynamic feeding conditions. Biotechnol Bioeng 87:145-60 (2004).

16. Dobroth ZT, Hu S, Coats ER, McDonald AG, Polyhydroxybutyrate synthesis on biodiesel wastewater using mixed microbial consortia. Bioresour Technol 102:3352-9 (2011).

17. Moita R, Freches A, Lemos PC, Crude glycerol as feedstock for polyhydroxyalkanoates production by mixed microbial cultures. Water Res 58:9-20 (2014).

18. Moralejo-Gárate H, Mar'atusalihat E, Kleerebezem R, van Loosdrecht MM, Microbial community engineering for biopolymer production from glycerol. Appl Microbiol Biotechnol 92:631-9 (2011). 
19. Vishniac W, Santer M, The Thiobacilli. Bacteriological Reviews 21:195-213 (1957).

20. APHA-AWWA-WPCF. Standard methods for the examination of water and wastewater. 21 st ed. Washington DC, USA: American Public Health Association/American Water Works Association/Water Environment Federation; 2005. 21. Smolders GJF, van der Meij J, van Loosdrecht MCM, Heijnen JJ, Stoichiometric model of the aerobic metabolism of the biological phosphorus removal process.

Biotechnol Bioeng 44:837-48 (1994).

22. Amann R, Krumholz L, Stahl DA, Fluorescent-oligonucleotide probing of whole cells for determinative, phylogenetic, and environmental-studies in microbiology. $J$ Bacteriol 172:762-70 (1990).

23. Loy A, Maixner F, Wagner M, Horn M, probeBase - an online resource for rRNA-targeted oligonucleotide probes: new features 2007. Nucleic Acids Res 35:D800D4 (2007).

24. Dionisi D, Majone M, Vallini G, Gregorio SD, Beccari M, Effect of the length of the cycle on biodegradable polymer production and microbial community selection in a sequencing batch reactor. Biotechnol Prog 23:1064-73 (2007).

25. Marang L, Jiang Y, van Loosdrecht MCM, Kleerebezem R, Impact of nonstoring biomass on PHA production: An enrichment culture on acetate and methanol. Int J Biol Macromol 71:74-80 (2014).

26. Dobrowolski A, Mituła P, Rymowicz W, Mirończuk AM, Efficient conversion of crude glycerol from various industrial wastes into single cell oil by yeast Yarrowia lipolytica. Bioresour Technol 207:237-43 (2016). 
27. Liu LP, Hu Y, Lou WY, Li N, Wu H, Zong MH, Use of Crude Glycerol as Sole Carbon Source for Microbial Lipid Production by Oleaginous Yeasts. Appl Biochem Biotechnol 182:495-510 (2016).

28. Jiang G, Hill DJ, Kowalczuk M, Johnston B, Adamus G, Irorere V, et al., Carbon Sources for Polyhydroxyalkanoates and an Integrated Biorefinery. Int J Mol Sci 17:1157-78 (2016).

29. Moralejo-Gárate H, Kleerebezem R, Mosquera-Corral A, Campos JL, PalmeiroSánchez T, van Loosdrecht MCM, Substrate versatility of polyhydroxyalkanoate producing glycerol grown bacterial enrichment culture. Water Res 66:190-8 (2014). 


\section{TABLE LEGENDS}

Table 1. Crude glycerol characterisation.

Table 2. Accumulation experiments with different carbon substrates using the enriched MMC collected from the SBR.

Table 3. Characterisation of accumulation experiments performed to test the maximum biopolymer accumulation with different substrates. 


\section{Table 1}

\begin{tabular}{lc}
\hline Parameter & \\
\hline $\mathrm{pH}$ & 10 \\
Density $\left(\mathrm{g} / \mathrm{L}, 20^{\circ} \mathrm{C}\right)$ & $966.9 \pm 7.2$ \\
Total Organic Carbon (TOC) $(\mathrm{g} \mathrm{C} / \mathrm{L})$ & $409.2 \pm 30.3$ \\
Glycerol (g/L) & $696.4 \pm 54.0$ \\
Methanol (g/L) & $225.4 \pm 22.9$ \\
$\mathrm{Lipids}^{(\mathrm{g} / \mathrm{L})}$ & $60.9 \pm 5.8$ \\
$\mathrm{Na}^{+}(\mathrm{g} / \mathrm{L})$ & $14.5 \pm 0.3$ \\
\hline
\end{tabular}


Table 2.

\begin{tabular}{|c|c|c|c|c|c|}
\hline \multirow{2}{*}{ Experiment } & \multirow{2}{*}{ Substrate } & \multirow{2}{*}{$\begin{array}{c}\text { Initial } \\
\text { biomass } \\
\text { (g VSS/L) }\end{array}$} & \multicolumn{3}{|c|}{ Initial substrate composition $(\mathrm{Cmmol})$} \\
\hline & & & Glycerol & $\mathrm{MeOH}$ & Lipids \\
\hline A1 & Crude glycerol & 0.52 & 180 & 120 & 140 \\
\hline A2 & Synthetic glycerol & 0.43 & 480 & 0 & 0 \\
\hline A3 & $\begin{array}{l}\text { Glycerol }+\mathrm{MeOH} \\
\text { (75:25 as } \mathrm{Cmmol})\end{array}$ & 0.45 & 370 & 110 & 0 \\
\hline A4 & $\begin{array}{l}\text { MeOH + Glycerol } \\
\text { (25:75 as Cmmol) }\end{array}$ & 0.55 & 120 & 320 & 0 \\
\hline
\end{tabular}


Table 3.

\begin{tabular}{|c|c|c|c|c|c|c|c|c|c|c|c|c|c|c|}
\hline \multirow{2}{*}{ Exp } & \multirow{2}{*}{$-q_{\text {gly }}$} & \multirow{2}{*}{$-q_{\mathrm{MeOH}}$} & \multirow{2}{*}{$q_{\text {TAG }}$} & \multirow{2}{*}{$q_{P H B}$} & \multirow{2}{*}{$q_{P H V}$} & \multirow{2}{*}{$q_{P G}$} & \multirow{2}{*}{$Y_{\text {TAG/TOC }}$} & \multirow{2}{*}{$\mathbf{Y}_{\mathrm{PHB} / \mathrm{gly}}$} & \multirow{2}{*}{$\mathbf{Y}_{\mathrm{PHV} / \mathrm{gly}}$} & \multirow{2}{*}{$\mathbf{Y}_{\mathrm{PG} / \mathrm{gly}}$} & \multicolumn{4}{|c|}{ Maximum (wt.\%) } \\
\hline & & & & & & & & & & & TAG & PHB & HPV & PG \\
\hline A1 & 0.43 & 0.13 & 0.40 & 0.00 & 0.03 & 0.14 & 0.52 & 0.00 & 0.06 & 0.33 & 46.3 & 0.0 & 7.4 & 27.6 \\
\hline A2 & 0.55 & --- & 0.05 & 0.02 & 0.04 & 0.18 & 0.08 & 0.02 & 0.06 & 0.31 & 11.4 & 3.6 & 14.4 & 54.3 \\
\hline A3 & 0.48 & 0.09 & 0.03 & 0.01 & 0.01 & 0.13 & 0.05 & 0.03 & 0.02 & 0.26 & 6.8 & 3.6 & 5.4 & 39.5 \\
\hline A4 & 0.25 & 0.17 & 0.00 & 0.02 & 0.00 & 0.02 & 0.00 & 0.08 & 0.00 & 0.04 & 3.9 & 8.6 & 2.1 & 8.2 \\
\hline
\end{tabular}

- $q_{\text {gly }}\left(\mathrm{Cmmol}\right.$ gly/Cmmol X.h); - $\mathrm{q}_{\mathrm{MeOH}}(\mathrm{Cmmol} \mathrm{MeOH} / \mathrm{Cmmol} X \cdot h) ; \mathrm{q}_{\mathrm{TAG}}(\mathrm{Cmmol} \mathrm{TAG} / \mathrm{Cmmol} \mathrm{X} \cdot \mathrm{h}) ; \mathrm{q}_{\mathrm{PHB}}(\mathrm{Cmmol} \mathrm{PHB} / \mathrm{Cmmol}$

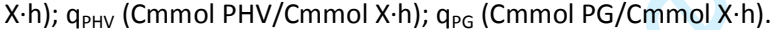

$\mathrm{Y}_{\mathrm{TAG} / \mathrm{TOC}}(\mathrm{Cmmol} \mathrm{TAG} / \mathrm{Cmmol} \mathrm{TOC}) ; \mathrm{Y}_{\mathrm{PHB} / \mathrm{gly}}(\mathrm{Cmmol} \mathrm{PHB} / \mathrm{Cmmol} \mathrm{gly}) ; \mathrm{Y}_{\mathrm{PHV} / \mathrm{gly}}\left(\mathrm{Cmmol} \mathrm{PHV/Cmmol} \mathrm{gly);} \mathrm{Y}_{\mathrm{PG} / \mathrm{gly}}(\mathrm{Cmmol}\right.$ PG/Cmmol gly).

wt.\% (\% g biopolymer/g cell dry weight). 


\section{FIGURE CAPTIONS}

Figure 1. Evolution of ammonium $(\bullet)$ and biomass $(O)$ concentration at the end of the cycles throughout the operation of the enrichment SBR.

Figure 2. FISH images of the biomass from the enrichment SBR on day 229. (A) Eukaryota domain (EUK516: Cy3, red) and Bacteria domain (EUB338mix: FITC, green). The bar represents $25 \mu \mathrm{m}$. (B) Betaproteobacteria class (BET42a: Cy3, red) and all DNA (DAPI, blue). The bar represents $10 \mu \mathrm{m}$.

Figure 3. Evolution of different compounds during an enrichment cycle on day 246 of operation. A) Glycerol $(\square)$, methanol $(\triangle)$, lipids $(\diamond)$ and TOC $(\bullet)$, all as $\mathrm{Cmmol}$, ammonium (x) as $\mathrm{Nmmol}$, and DO (-) as mg $\left.\mathrm{O}_{2} / \mathrm{L} . \mathrm{B}\right) \operatorname{TAG}(\bullet), \operatorname{PHV}(\mathrm{O}), \mathrm{PG}(\square)$ and active biomass (- - -), all as $\mathrm{Cmmol}$.

Figure 4. Evolution of different compounds throughout accumulation assays with different substrates. A) Crude glycerol-A1; B) Synthetic glycerol-A2; C) Mix of synthetic glycerol and methanol (75:25 ratio as Cmmol)-A3; D) Mix of synthetic glycerol and methanol (25:75 ratio as $\mathrm{Cmmol})$-A4. Measurements of glycerol $(\boldsymbol{\square})$, methanol $(\triangle)$, lipids $(\diamond)$, TOC $(\bullet), \operatorname{TAG}(\bullet), \operatorname{PHB}(\mathbf{\Delta}), \operatorname{PHV}(O), \operatorname{PG}(\square)$, active biomass $(---)$, all as Cmmol. 


\section{Figure 1}

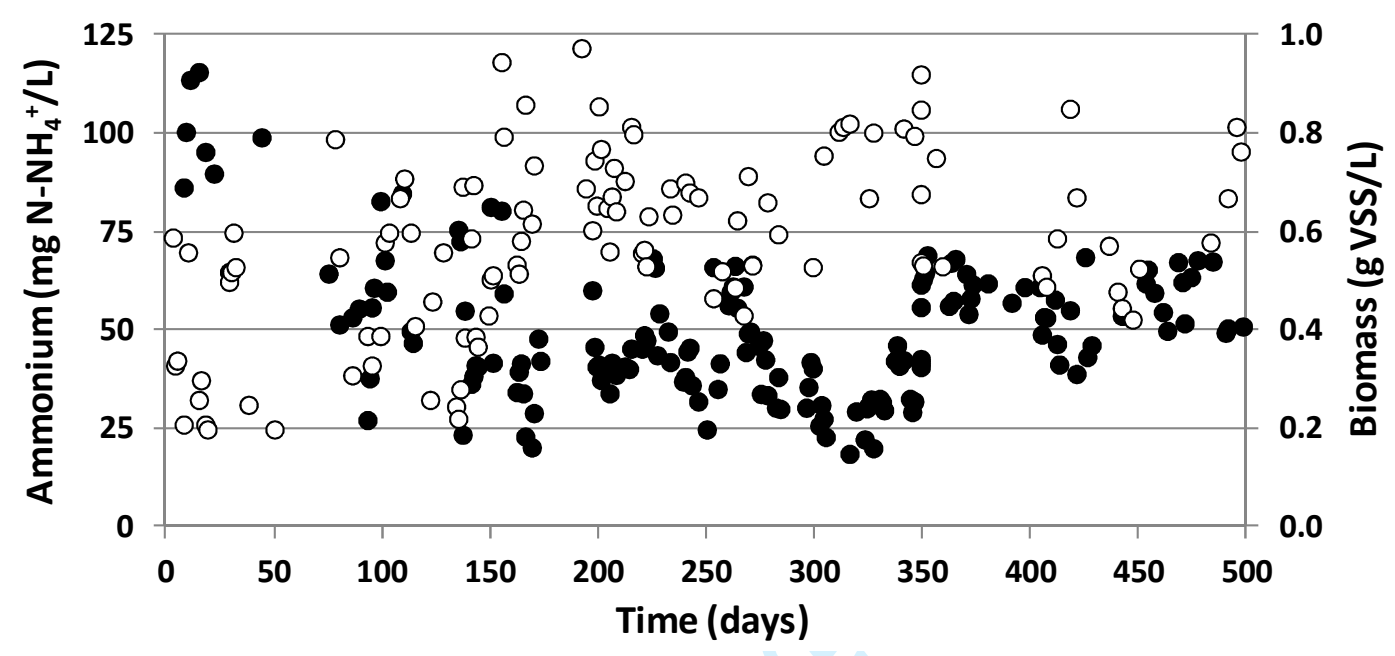


Figure 2

A

B
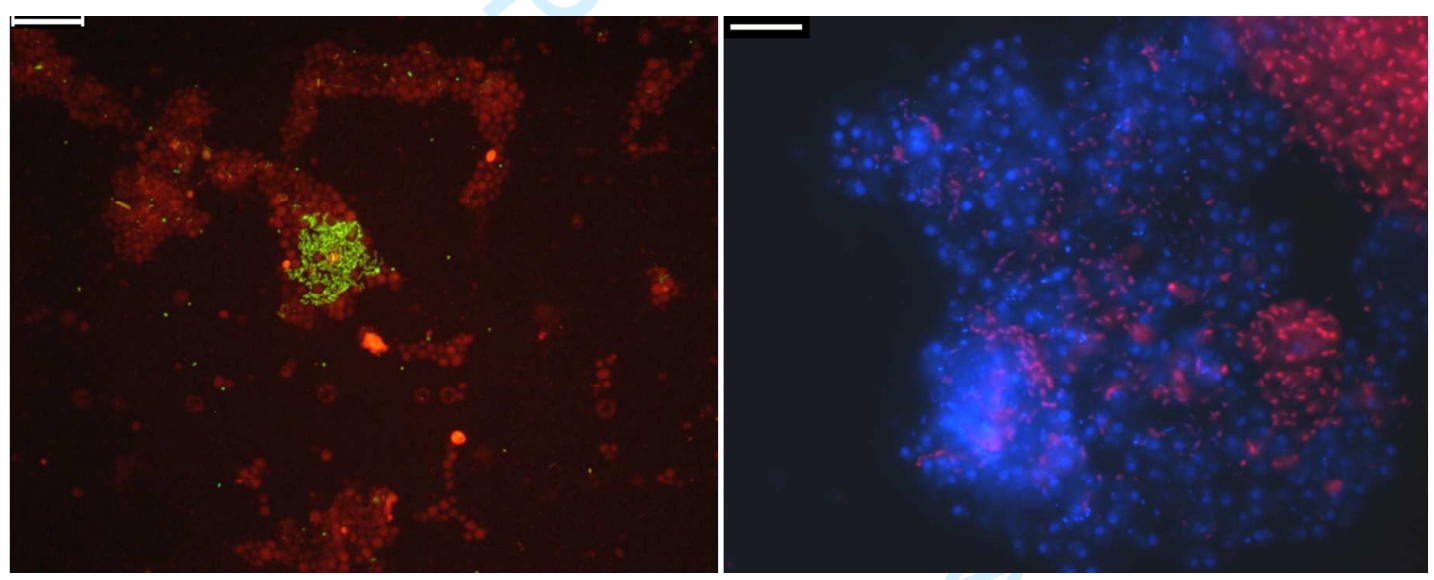


\section{Figure 3}

A

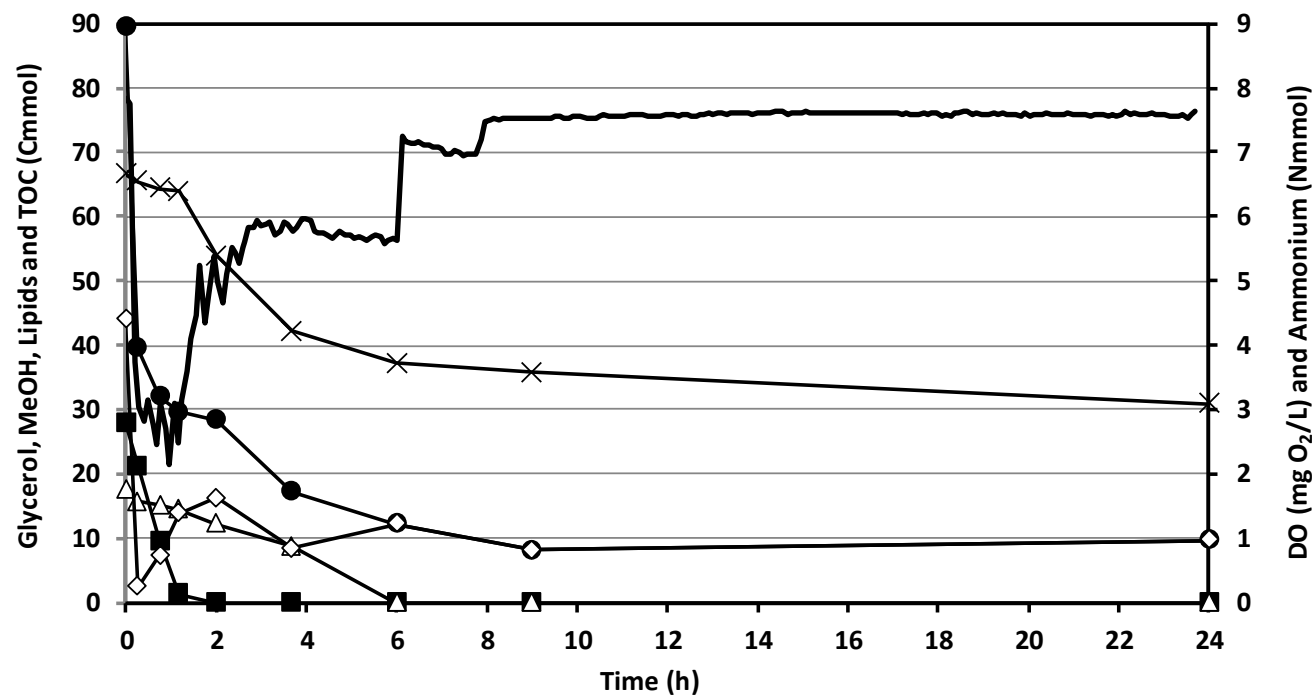

B

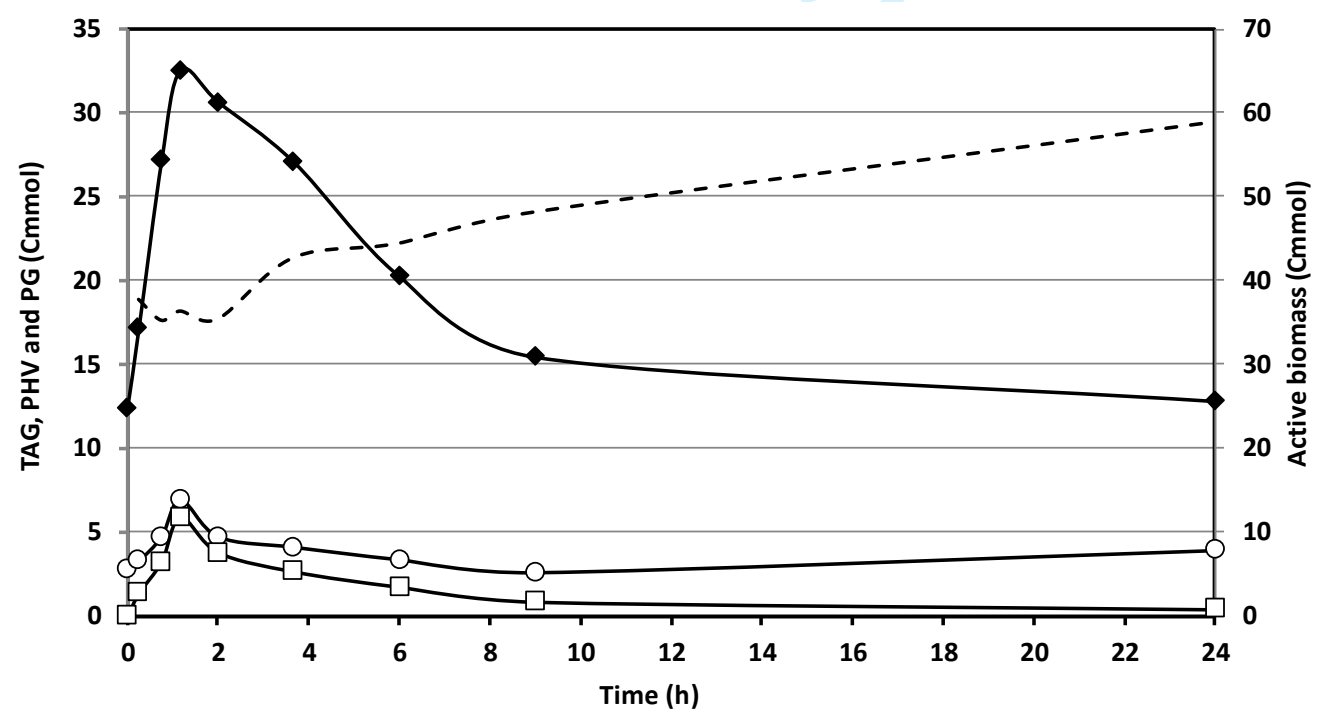

http://mc.manuscriptcentral.com/jctb-wiley 
Figure 4

A

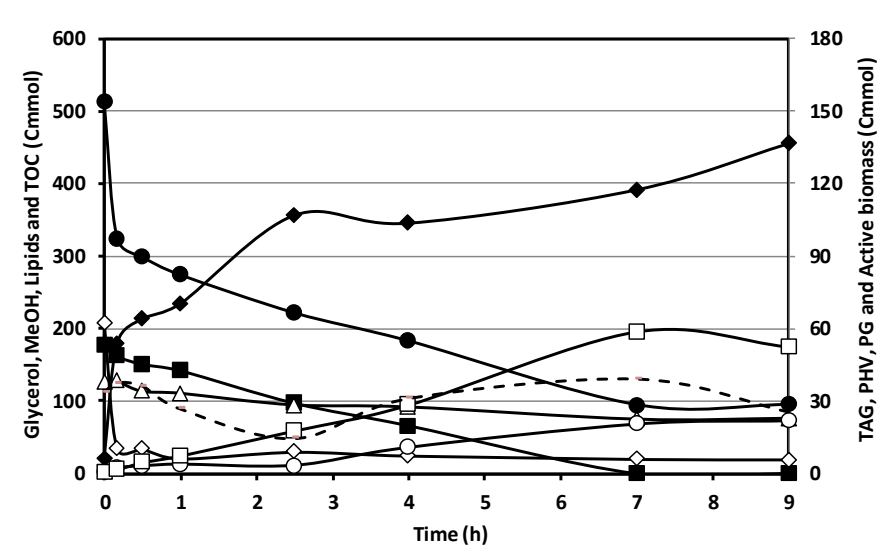

C

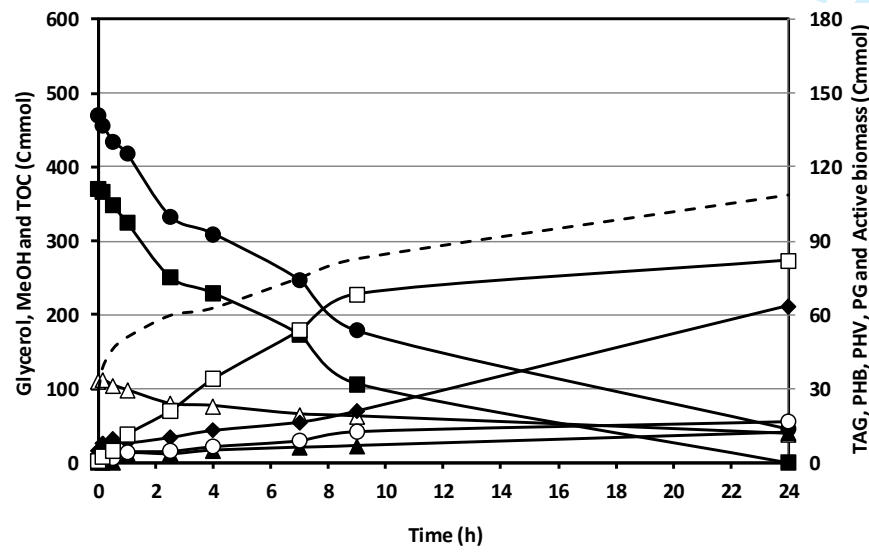

B

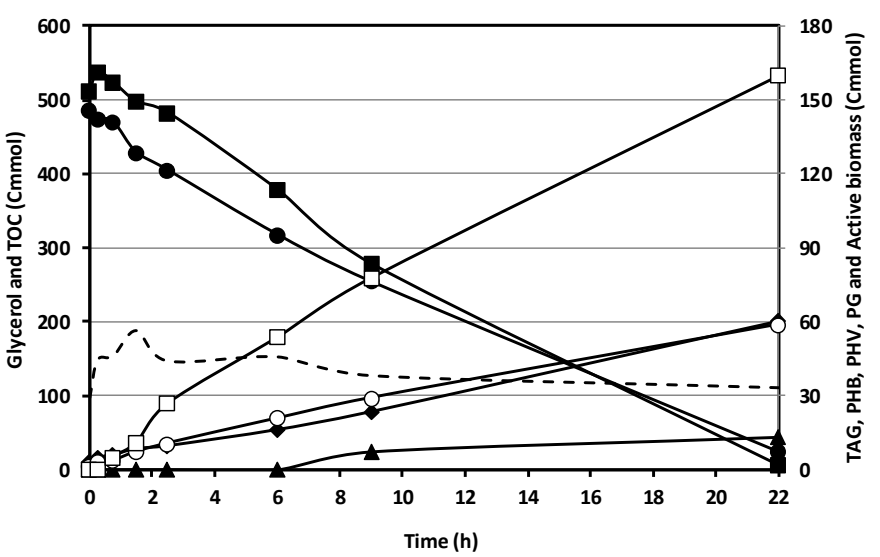

D

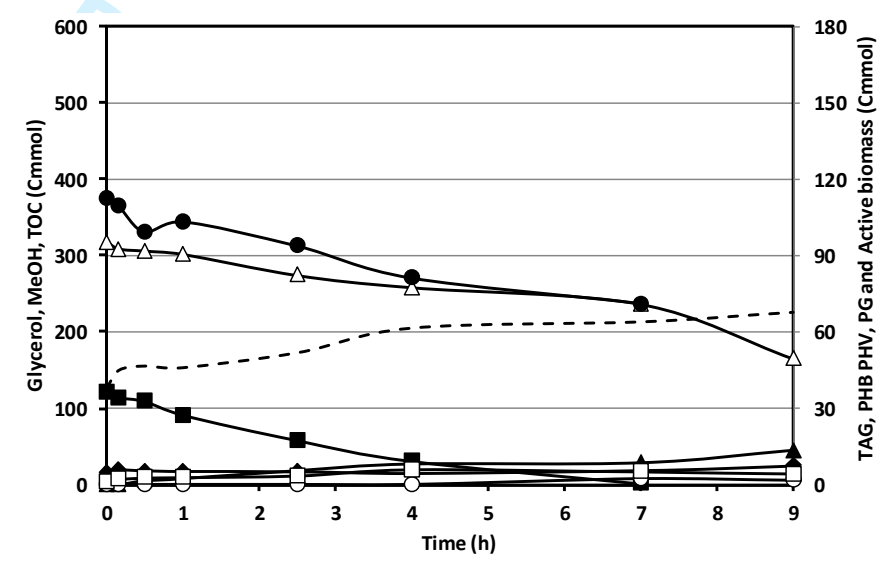


SUPPLEMENTARY MATERIAL

Table S1. List of oligonucleotide probes used for biomass FISH analysis, formamide percentages and identified microorganisms.

\begin{tabular}{|c|c|c|}
\hline Probe & \% Formamide & Target organism \\
\hline EUB338I & $0-50$ & Bacteria domain \\
\hline EUB338II & $0-50$ & Planctomycetales \\
\hline EUB338III & $0-50$ & Verrucomicrobia \\
\hline BET42a & 35 & Betaproteobacteria class \\
\hline EUK516 & 25 & Eukarya domain \\
\hline
\end{tabular}

EUB338I, EUB338II and EUB338III were applied as an equimolar mix (EUB338mix)

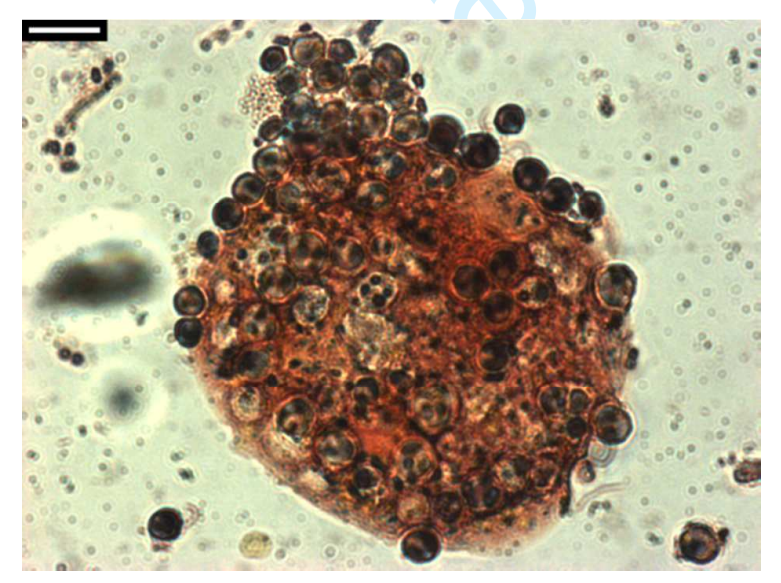

Figure S1. Biomass sample from the enrichment SBR collected during the feast phase on day 478 and treated with Sudan staining to observe the biopolymer inclusions inside the yeast cells. The bar represents $10 \mu \mathrm{m}$. 